\title{
Proceedings of the 50th anniversary meeting of the Association of British Neurologists in London, 4-5 November 1983.
}

THE VALUE OF A ROUTINE CT SCAN AND A CARDIAC OPINION IN THE ASSESSMENT OF ACUTE STROKE

PAG Sandercock, CP Warlow, IR Starkey, A Molyneaux

We examined data on 296 consecutive cases of first stroke (diagnosed by clinical criteria) in our community based stroke register to determine whether CT scanning and echocardiography should be performed routinely. 248/296 (84\%) cases had a CT scan, but only five of these showed a non-vascular lesion. There were a further 108 patients referred by general practitioners as possible cases of stroke, in whom CT scanning detected nine spaceoccupying lesions; in all nine cases our clinical assessment had suggested that stroke was highly unlikely. CT scanning is said to have a particular use in the diagnosis of cerebellar haemorrhage; in our series only five cases were found but four had died before CT scanning could be undertaken. There were 39 patients $(13 \%)$ in atrial fibrillation; six proved to have primary intracerebral haemorrhages, or haemorrhagic cerebral infarcts. Patients aged under 76 (years) with a cerebral infarct not due to arteritis or blood dyscrasia (108/291 first strokes) were to be assessed prospectively by a cardiologist. $98 / 108(91 \%)$ were available for clinical assessment, and 92/98 (94\%) for echocardiography. The cardiologist's clinical assessment altered the neurologist's cardiac diagnosis in $8 / 98(8 \%)$. The echocardiogram proved useful in the further assessment of the 13 patients with atrial fibrillation, and eight patients with clinically diagnosed valvular lesions, but detected only 10 clinically unsuspected cardiac lesions of uncertain relevance. CT scanning and echocardiography have a place in the diagnosis of acute stroke, but only in a small proportion, who can be selected by simpler criteria.

DOPPLER ULTRASONOGRAPHY IN THE INVESTIGATION OF CAROTID ARTERY DISEASE PRD Humphrey, PG Bradbury

Recent evidence suggests that the proportion of patients undergoing angiography for the investigation of transient ischaemic attacks (TIA), varies from 4 to $100 \%$ in different centres around Britain. Over the past two years nearly 500 Doppler examinations of the extracranial carotid and vertebral arteries have been performed at the National Hospital. Approximately 70 of these patients have subsequently come to angiography. This paper compares the results of the Doppler and angiographic studies. There was complete agreement between the two methods in the 16 patients with carotid occlusion. In those with carotid stenosis (30-99\% narrowing of the lumen) the Doppler technique correctly diagnosed 27 out of the 31 cases. In those with mild atheroma (up to $30 \%$ narrowing of the lumen), there was agreement in only 10 of the 19 patients. Finally in the 40 studies with normal angiographic bifurcations, the Doppler was correct in 37 . These results indicate that Doppler examination is useful in the assessment of carotid occlusions and stenoses but the technique is of limited use in the detection of minor atheromatous disease. Such patients are, however, usually treated medically, angiography being indicated only in those few patients considered to have stenoses amenable to surgery. The equipment is inexpensive but requires some expertise to use. Once mastered, the technique provides a reasonably accurate method for screening patients under consideration for angiography, thereby avoiding unnecessary angiographic studies. It may also detect significant but clinically silent lesions.

PROSTAGLANDIN CHANGES IN ISCHAEMIC CEREBRAL TISSUE

HA Crockard, KK Bhakoo, PT Lascelles Tissue prostaglandins of the $F_{2 \alpha}$ and $E_{i}$ series together with fatty acid precursors have been assayed in gerbil brain subjected to profound transient global ischaemia The changes have been correlated with measurements of oedema and alterations in blood brain barrier permeability. During ischaemia, there is a modest increase in brain water content and a rise in PG $F_{2 \alpha}$; with reperfusion there is a thirty-fold increase which subsides within three hours.
$\mathrm{PGE}_{2}$ levels do not rise until the end of the first hour of reperfusion and coincide with evidence of increased blood brain barrier permeability. The fatty acid precursors of prostaglandins also increase with ischaemia and during the initial phase of reperfusion. There is a non-specific rise in all fatty acids, but these are not affected by blockade of the phospholipase $A_{2}$ system. We conclude that ischaemia produces a non-specific breakdown of cell membranes, and within brain tissue products are metabolised along the $\mathrm{PGF}_{2 \alpha}$ pathway. $\mathrm{PGE}_{2}$ may arise from the intravascular compartment and may be involved in the process of altered blood brain barrier permeability.

INHIBITORY MECHANISMS IN THE VISUAL CORTEX

KAC Martin, P Somogyi, D Whitteridge We have looked at the physiologicalo properties of single nerve cells in the visual cortex and then filled them intracellularlyô with horseradish peroxidase. So far we have recovered 125 such cells of which $65 \%$ are pyramidal, $20 \%$ spiny stellate and $13 \%$ are smooth stellate. We have concentrated on this last group which is responsible for all cortical inhibition. The most frequent type are large basket cells of which we have seen six and studied three in detail. They are responsible for the GABA staining baskets round the perikarya of pyramidal cells, but each basket cell only sends one or at most two axonal branches each with about five boutons to every one perikaryon. It takes axons from 15-20 such basket cells to make up the "basket" on each pyramidal perikaryon. The basket cells also send $50 \%$ of their endings to dendrites ending on spines and on shafts. There is some concentration of endings on the origins of the apical dendrites and the basal dendrites. The most remarkable cell is the "chandelier" cell whose axons almost exclusively supply a group of endings around the initial segment of the axon Clearly excitatory and inhibitory effects do not simply add, but the effectiveness of inhibitory boutons is increased by their strategic placement. 
A STUDY OF LYMPHOCYTES SUBPOPULATIONS IN MULTIPLE SCLEROSIS FAMILIES

DAS Compston, PJ Hughes

Non-specific cytotoxic/suppressor lymphocytes (OKT8 positive) are periodically reduced in peripheral blood of patients with multiple sclerosis (MS), particularly during relapse or in the progressive phase, providing further evidence for an immunological abnormality in the disease. Amongst other possibilities, this change in immuno-regulatory cells could be an expression of genetic susceptibility to MS or the consequence of environmental factors involved in the pathogenesis. We have made serial measurements of circulating OKT8 and OKT4 (helper/inducer) positive cells at fortnightly intervals for 6 months in nine unrelated patients with chronic progressive MS, six of their spouses, 14 siblings and 10 unrelated healthy individuals. Significant reductions in OKT8 cells were frequently found in the patients, their spouses and some siblings, but rarely in the normal controls. These findings are consistent with the hypothesis that environmental factors lead to abnormal lymphocyte subpopulations in patients with MS.

DELAYED HYPERSENSITIVITY REACTIONS IN PERIPHERAL NERVE WITH AND WITHOUT AN IMMUNE RESPONSE TO GALACTOCEREBROSIDE RAC Hughes, HC Powell, PW Lampert

It had been suggested that non-specific immune responses in the nervous system might be an important mechanism in demyelinating disease. We therefore immunised guinea pigs and rabbits with Freund's complete adjuvant and four weeks later challenged them by injecting 5 or $10 \mu \mathrm{l}$ tuberculin $(2 \mathrm{mg} / \mathrm{ml})$ into their sciatic nerves. Six days later the sciatic nerves were removed and embedded in plastic for electron microscopy. Although there was extensive inflammatory cell infiltration and some Wallerian degeneration, primary demyelination was insignificant. Rabbits immunised with complete adjuvant, the myelin lipid hapten galactocerebroside and bovine albumin as a carrier, developed high titres of complement fixing antibody to galactocerebroside which were largely directed against the galactose portion of the molecule. A tuberculin reaction in the sciatic nerves of these animals induced extensive primary demyelination. We conclude that a nonspecific immune response in the nervous system can elicit demyelination in an animal which is already sensitised to a myelin or Schwann cell surface antigen. (Sup- ported by the Kroc Foundation, the NINCD (NS 14162), and a MRC Travelling Research Fellowship to RACH).

NMR IMAGING OF POSTERIOR FOSSA TUMOURS DJ Thomas, RD Hayward, J Marshall, G Bydder

NMR with its better white/grey contrast on $T_{1}$ scans has been shown to be superior to $\mathrm{X}$-ray CT in detecting lesions in multiple sclerosis. Its advantages in tumour identification have been less certain. The two methods were therefore compared in 26 patients with tumours in the posterior fossa. X-ray CT was performed on a Siemens Somatom, two with contrast enhancement, except in patients with known iodine sensitivity. $T_{1}$ and $T_{2}$ images were obtained on a Thorn EMI NMR scanner without enhancement. Seven patients had benign tumours. These were shown equally well in three cases but NMR was superior in four. NMR detected all 19 cases with malignant tumours. Two were missed on X-ray CT. Three cases were shown equally well. CT was superior in two cases and then only after contrast. Ventricular size was equally well shown but periventricular oedema was better shown on NMR. Bony erosion was demonstrated more satisfactorily on CT. NMR is still in the early stages of its development. Further improvements are still to be expected with modified sequences and the use of enhancement techniques. Nevertheless its advantages over conventional CT in tumour detection have been demonstrated.

NEUROLOGICAL ASSESSMENT OF THE NEWBORN INFANT

Victor Dubowitz

The traditional neurological approach is not very practical in trying to assess the functional state of the nervous system in a newborn infant. In addition to trying to define abnormality one also has to take account of the physiological changes occurring with maturation of the nervous system. A system of examination has been devised incorporating a series of neurological signs (including motility, tone and primitive reflexes) and neurobehavioural responses (including visual and auditory orientation and response decrement). Each individual item is scored on a 5-point scale and recorded directly on the instruction/ recording sheet. The overall pattern of the responses is visualised and deviant signs can be readily recognised. The system has proved practical in both premature and full-term infants and can also be used sequentially in following the progress of individual infants. In a correlative study of 100 consecutive infants in whom the presence of periventricular haemorrhage (PVH) was diagnosed in the newborn period by ultrasound examination, independent sequential clinical assessment identified 5 signs which correlate with the presence of PVH. Follow-up studies (up to 1 year to date) have shown a good correlation with the predicted outcome based on the examinations in the neonatal period. The method has proved to be practical and objective and should provide a useful basis for the routine assessment of neurological status of the newborn infant.

A LONG TERM RANDOMISED COMPARISON OF VALPROATE AND PHENYTOIN IN ADULT PATIENTS WITH EPILEPSY

DM Turnbull, MD Rawlins, D Chadwick A long term study comparing sodium valproate and phenytoin monotherapy in the treatment of adult onset epilepsy has been undertaken. This included 140 patients, 76 presenting with tonic-clonic seizures with no clinical evidence of a focal component, and 64 with complex partial or simple partial seizures with or without secondary generalised seizures. Of these 140 patients, 18 patients were lost to follow up or were consistently found not to be taking their medication. All other patients were followed up for between 2 and 4 years. The results of the study were analysed by a log ranked statistical method; the parameters of success used were time to 1 year remission, time to 2 year remission and time to first seizure. When comparing the two treatment groups, there was no significant difference between phenytoin and valproate monotherapy in the treatment of either tonic-clonic $(p=0.2)$ or partial $(p=0.53)$ seizures. Combining both treatment groups, we have also studied the factors likely to impair the prognosis in patients presenting with adult onset epilepsy. The only factor which influenced the time to 2 year remission was seizure type, with a significantly poorer response in patients with partial seizures $(p=0 \cdot 001)$. In terms of time to first seizure there was, in addition to seizure type, a significant difference between those with low and high pretreatment frequencies of partial seizures $(p=$ $0 \cdot 0004$ ). Idiosyncratic adverse effects were more common in the phenytoin treatment group (five patients compared with no patients in the valproate group) but this difference was not significant $(p=0 \cdot 06)$. 
THE VALUE OF ROUTINE CT SCANNING AND ECHOCARDIOGRAPHY IN THE DIAGNOSIS OF ACUTE STROKE

PAG Sandercock, CP Warlow, IR Starkey, A Molyneaux

PRESIDENTIAL ADDRESS: THE HISTORY OF THE ASSOCIATION

PIC Robinson

PRESENT CONCEPTS OF ORGANISATION AND FUNCTION OF THE CEREBRAL CORTEX

G Baumgartner

(the first Gordon Holmes Lecture)
INFECTION AND DEGENERATIVE DISEASE OF THE NERVOUS SYSTEM

WB Matthews

THE CEREBROSPINAL FLUID AS AN INDEX OF THE PATHOPHYSIOLOGY OF THE BRAIN AN Davison

THE EFFECT OF INJURY ON SENSORY NERVE ENDINGS AND ON NERVE FIBRES PD Wall
Proceedings

AUTONOMIC DISORDERS OF THE PELVIC ORGANS

GS Brindley

DISORDERS OF THE CAROTID ARTERY

R Ross Russell

CHANGING CONCEPTS OF PERIPHERAL NERVE DISEASE 1933-1983

RW Gilliatt

EXTRAORDINARY COMMUNICATION: THE ROLE OF THE NEUROLOGIST

A Hopkins 